\title{
An empirical pipeline for determining the viscosity parameter for Be star disks
}

\author{
Leandro R. Rímulo ${ }^{1}$, Alex C. Carciofi ${ }^{1}$, Thomas Rivinius ${ }^{2}$ and \\ Xavier Haubois ${ }^{3}$ \\ ${ }^{1}$ University of São Paulo, Dept. of Astronomy, Brazil \\ email: lrrimulo@usp.br \\ ${ }^{2}$ ESO, Chile \\ ${ }^{3}$ LESIA, Observatoire de Paris, France
}

\begin{abstract}
Be star phenomenology is strongly associated with their viscous circumstellar disks. Recently, models became available for the temporal evolution of these disks when subject to variable mass ejection rates. In this contribution we will discuss how these dynamical disk models, modeled with the radiative transfer code HDUST, can be used for constraining fundamental disk parameters, such as the $\alpha$ viscosity parameter, and we will report on an ongoing effort to model light curves of a large number of stars.
\end{abstract}

Keywords. hydrodynamics, radiative transfer, techniques: photometric, stars: mass loss

\section{Introduction}

Be stars are non supergiant rapid rotating B stars $\left(3\right.$ to $\left.15 M_{\odot}\right)$, whose spectrum has, or had at some time, Balmer lines in emission, attributed to a geometrically thin circumstellar gaseous decretion disk. Roughly $10 \%$ of the B stars in the Galaxy are Be stars, and this percentage grows for the Magellanic Clouds, where metallicity is lower (Martayan et al. 2011). The circumstellar disk is created by mass ejected from the rotating stellar surface to the disk, which then diffuses outwards by turbulent viscosity, as observational and theoretical studies have shown. Hence their name "viscous decretion disks" (VDD) (see review by Rivinius et al. 2013).

The VDD scenario in the steady state limit has been successfully tested for several Be stars, using the radiative transfer code HDUST (e.g., Carciofi \& Bjorkman 2006; Carciofi et al. 2007, 2009, 2012). However, there are still several open theoretical questions for the VDD scenario, one of the main ones being the origin and magnitude of the turbulent viscosity. Measuring directly (or as directly as possible) the value of Shakura-Sunyaev's $\alpha$ viscosity parameter is of great current interest.

Variability is one of the main observational features of Be stars. They are attributed to a variable mass injection rate from the star to the disk. Using the 1-D time-dependent hydrodynamic code SINGLEBE (Okazaki 2007) associated with the radiative transfer code HDUST (Carciofi \& Bjorkman 2006, 2008), it was possible to determine, for the first time, $\alpha=1.0 \pm 0.2$ for a dissipation phase of the disk of the Be star $28 \mathrm{CMa}$ (Carciofi et al. 2012). Currently, using the cited codes, we are developing a simulation based chain of procedures (a pipeline) aimed to estimate the $\alpha$ parameter, for the first time, for hundreds of light curves available from photometric surveys (e.g., OGLE, MACHO, EROS). 


\section{Modeling dynamical viscous decretion disks}

From the $\alpha$-disk formalism (Shakura \& Sunyaev 1973), the equation for the evolution of the distribution of mass in the disk as function of radial distance $R$ and time $t$ is

$$
\frac{\partial \Sigma}{\partial t}=\frac{1}{\tau_{\text {vis }}}\left\{\frac{2}{r} \frac{\partial}{\partial r}\left[r^{\frac{1}{2}} \frac{\partial}{\partial r}\left(r^{2} \Sigma\right)\right]+\left(\frac{r_{\text {in }}^{2}}{r_{\text {in }}^{\frac{1}{2}}-1} \frac{\delta\left(r-r_{\text {in }}\right)}{r_{\text {in }}}\right) \Sigma_{\text {in }}(t)\right\}
$$

where $r=R / R_{\star}, r_{\text {in }}=R_{\text {in }} / R_{\star}\left(R_{s}\right.$ being the radius of mass injection to the disk).

The constant $\tau_{\mathrm{vis}}$ is given by $\frac{1}{\tau_{\mathrm{vis}}}=\alpha \frac{k T}{\mu m_{H}\left(G M R_{\star}\right)^{\frac{1}{2}}}$. Therefore, the greater the viscosity parameter $\alpha$, the faster is the evolution of the disk surface density. The variable $\Sigma_{\text {in }}(t)$ parameter is a more appropriate form of stating the mass injection rate $-\dot{M}_{\mathrm{inj}}(t)$. Both are related by: $2 \pi R_{\star} \Sigma_{\mathrm{in}}(t)\left(\frac{R_{\star}}{\tau_{\mathrm{vis}}}\right)=-\dot{M}_{\mathrm{inj}}(t)\left(\frac{r_{\mathrm{in}}^{\frac{1}{2}}-1}{r_{\mathrm{in}}^{2}}\right)$.

\subsection{The pipeline}

We found that the light curves associated with a disk dissipation following a disk construction that lasted a time $\Delta t$ (disk build-up time) assume the following empirical law

$$
m_{\lambda}(t)=m_{(t=\infty), \lambda}-\left(\Delta m_{\lambda}\right) e^{-\left(\xi_{\lambda} \frac{t}{\tau_{\text {vis }}}\right)}
$$

valid in general, for non-edge-on cases: $i<60^{\circ}$ (edge-on cases being observationally identifiable by their behavior in color), where $\lambda$ denotes a given photometric band.

The limiting magnitude, $m_{(t=\infty), \lambda}$, depends only on the stellar parameters. In order to calibrate empirically $\xi_{\lambda}$ and $\Delta m_{\lambda}$ we set up a very large model grid consisting of several spectral types for the central star (from B0 to B9), $\Sigma_{\text {in }}$ (roughly from 0.1 to $4 \mathrm{~g} \mathrm{~cm}^{2}$ ), $\Delta t$ (from 0.1 to 5 years) and $i$ (from $0^{\circ}$ to $65^{\circ}$ ).

We summarize our pipeline for finding $\alpha$ from light curves as follows:

(a) Find a "bump like" light curve of a Be star with known distance and interstellar absorption law, with a few years of coverage, and two color photometry;

(b) Exclude the curve if it corresponds to a near edge-on case;

(c) Find a good approximation to the stellar parameters $R_{\star}, T_{\text {eff }}$ and $M$ by measuring the limiting magnitude $m_{(t=\infty), \lambda}$ in two filters;

(d) Measure the build-up interval $\Delta t$ and the excess $\Delta m_{\lambda}$ directly from the light curve. Then, with our tabulated values, find the asymptotic surface density $\Sigma_{\text {in }}$. And with it and our tabulated values again, find the $\xi_{\lambda}$ parameter;

(e) Fit our derived law given by Eq.(2.2) on the dissipation part of the curve and obtain the coefficient of the exponential: $\left(\xi_{\lambda} / \tau_{\text {vis }}\right)_{\text {fitted }}$. Then, obtain, the $\alpha$ viscosity parameter: $\alpha=\left(\xi_{\lambda} / \tau_{\text {vis }}\right)_{\text {fitted }} /\left(\xi_{\lambda} / \alpha \tau_{\text {vis }}\right)_{\text {pipeline }}$.

\section{References}

Carciofi, A. C. \& Bjorkman, J. E. 2006, ApJ 639, 1081

Carciofi, A. C. \& Bjorkman, J. E. 2008, ApJ 684, 1374

Carciofi, A. C., Bjorkman, J. E., Otero, S. A., et al. 2012, ApJ (Letters) 744, id. L15

Carciofi, A. C., Magalhães, A. M., Leister, N. V., Bjorkman, J. E., \& Levenhagen, R. S. 2007, ApJ (Letters) 671, L49

Carciofi, A. C., Okazaki, A. T., Le Bouquin, J.-B., et al. 2009, A\&\&A 504, 915

Martayan, C., Rivinius, T., Baade, D., Hubert, A.-M., \& Zorec, J. 2011, IAU Circ. 272, 242

Okazaki, A. T. 2007, ASP-CS 361, 230

Rivinius, T., Carciofi, A. C., \& Martayan, C. 2013, A\&A Rev. 21, id.69

Shakura, N. I. \& Sunyaev, R. A. 1973, A\&A 24, 337 